\title{
Úlcera de Lipschütz, un diagnóstico para considerar en la población pediátrica
} Ulcer of Lipschütz, a diagnosis to consider in the pediatric population

\author{
Dr. Javier Arellano ${ }^{a, b}$, Lic. Eliseo Fuentes ${ }^{c}$, Dra. Patricia Moreno ${ }^{d}$ y Dra. Yamile Corredoira ${ }^{e}$
}

\begin{abstract}
RESUMEN
La úlcera vulvar aguda o úlcera de Lipschütz corresponde a una entidad poco frecuente, por lo general, subdiagnosticada. Se caracteriza por la aparición súbita o aguda de lesiones ulcerosas y dolorosas en la vulva, la vagina y/o el periné, sin antecedente de contacto sexual en niñas y adolescentes. Su etiología permanece desconocida en la mayoría de los casos, aunque se ha asociado a agentes infecciosos. Para su estudio, se deben sospechar infecciones de transmisión sexual, reacciones adversas a medicamentos, enfermedades autoinmunes e inmunosupresión. Las lesiones ulcerosas desaparecen espontáneamente, de manera habitual, sin secuelas ni recurrencias a largo plazo. Se presenta el caso de una paciente adolescente de 11 años, sin inicio de actividad sexual ni menarquia y que consultó por un cuadro prodrómico de cuatro días, seguido del desarrollo agudo de úlceras genitales. Se discuten sus causas, presentación clínica, diagnósticos diferenciales y tratamiento.

Palabras clave: úlcera de Lipschütz, enfermedad genital femenina, aguda.
\end{abstract}

\begin{abstract}
Acute vulvar ulcer or ulcer of Lipschütz corresponds to a rare disease, being usually underdiagnosed. It is characterized by a sudden or acute development of ulcerous and painful lesions in the vulva, vagina and/or perineum, without a previous history of sexual contact in girls and teenagers. Its etiology remains unknown in most cases, although they have been associated with infectious agents. Their study includes the suspicion of sexually transmitted infections, adverse drug reactions, autoimmune diseases and immunosuppression. The
\end{abstract}

a. Departamento de Dermatología, Sede Centro, Facultad de Medicina, Universidad de Chile.

b. Servicio de Dermatología, Hospital Clínico San BorjaArriarán

c. Facultad de Medicina, Universidad de Chile.

d. Universidad de Chile.

e. Departamento de Anatomía Patológica, Sede Centro, Facultad de Medicina, Universidad de Chile.

Santiago, Chile.

Correspondencia:

Dr. Javier Arellano: drjavierarlo@gmail.com

Financiamiento: Ninguno.

Conflicto de intereses: Ninguno que declarar.

Recibido: 28-8-2018

Aceptado: 8-1-2019 ulcerative lesions disappear spontaneously, usually without sequelae or recurrences in the long term. We present the case of an 11-year-old adolescent patient, without sexual initiation, or menarche and who consulted due to a four-day prodromal condition, followed by the acute development of genital ulcers. Its causes, clinical presentation, differential diagnosis and treatment are discussed.

Key words: Lipschütz ulcer, genital disease female, acute.

http: / / dx.doi.org/10.5546/ aap.2019.e305

Cómo citar: Arellano J, Fuentes E, Moreno P, Corredoira Y. Úlcera de Lipschütz, un diagnóstico para considerar en la población pediátrica. Arch Argent Pediatr 2019;117(3):e305-e308.

\section{INTRODUCCIÓN}

Las úlceras genitales presentan una etiología diversa con múltiples agentes infecciosos involucrados. Los más prevalentes a nivel mundial son las infecciones de transmisión sexual, específicamente, causadas por el virus herpes simple (VHS). ${ }^{1}$ En cambio, la úlcera de Lipschütz es la causa más frecuente en la población pediátrica, la cual corresponde a una patología de etiología desconocida e infrecuente que afecta, sobre todo, a niñas y a adolescentes sin contacto sexual previo. $^{2}$ Se caracteriza por el desarrollo agudo de úlceras vulvovaginales, que se pueden asociar, durante el desarrollo de las lesiones, a síntomas prodrómicos..$^{2-4}$

Las lesiones pueden debutar como vesículas eritematovioláceas, que, en su totalidad, evolucionan a úlceras dolorosas de bordes irregulares y fondo necrótico, y presentan una resolución espontánea en 2 o 3 semanas, sin tendencia a la recidiva ni cicatrices. ${ }^{2-5} \mathrm{Su}$ diagnóstico es esencialmente clínico, y se reserva la biopsia de la piel para casos particulares, como duda diagnóstica, sospecha de abuso sexual o malignidad, tanto por lo traumático del procedimiento como por la edad del paciente. ${ }^{4-7}$

El tratamiento de esta enfermedad, en los casos leves, es sintomático y, en los casos moderados, incluye corticoides tópicos. En los casos graves, persistentes o refractarios, debe considerarse la hospitalización para el tratamiento antibiótico 
oral o endovenoso, corticoides sistémicos y la eventual debridación quirúrgica..$^{7-10}$

Se presenta a una adolescente de 11 años de edad con diagnóstico de una úlcera de Lipschütz, con clínica e histología compatible.

\section{CASO CLÍNICO}

Paciente de sexo femenino, de 11 años y 4 meses de edad, sin antecedentes personales patológicos, sin uso de medicación, sin menarquia ni inicio de actividad sexual. No presentaba antecedentes familiares de relevancia.

Consultó por un cuadro de 4 días de evolución caracterizado por cefalea, náuseas, anorexia, astenia, adinamia y fiebre $\left(39^{\circ} \mathrm{C}\right)$, y se agregó dolor vulvovaginal y desarrollo de lesiones vesiculares eritematosas. Evaluada ambulatoriamente el segundo día del cuadro clínico, con diagnóstico presuntivo de infección herpética, fue tratada con aciclovir y mupirocina con pobre respuesta clínica, por lo cual se decidió hospitalizar.

FIGURA 1. Úlceras de bordes netos en la zona mucosa vulvar y en el labio mayor

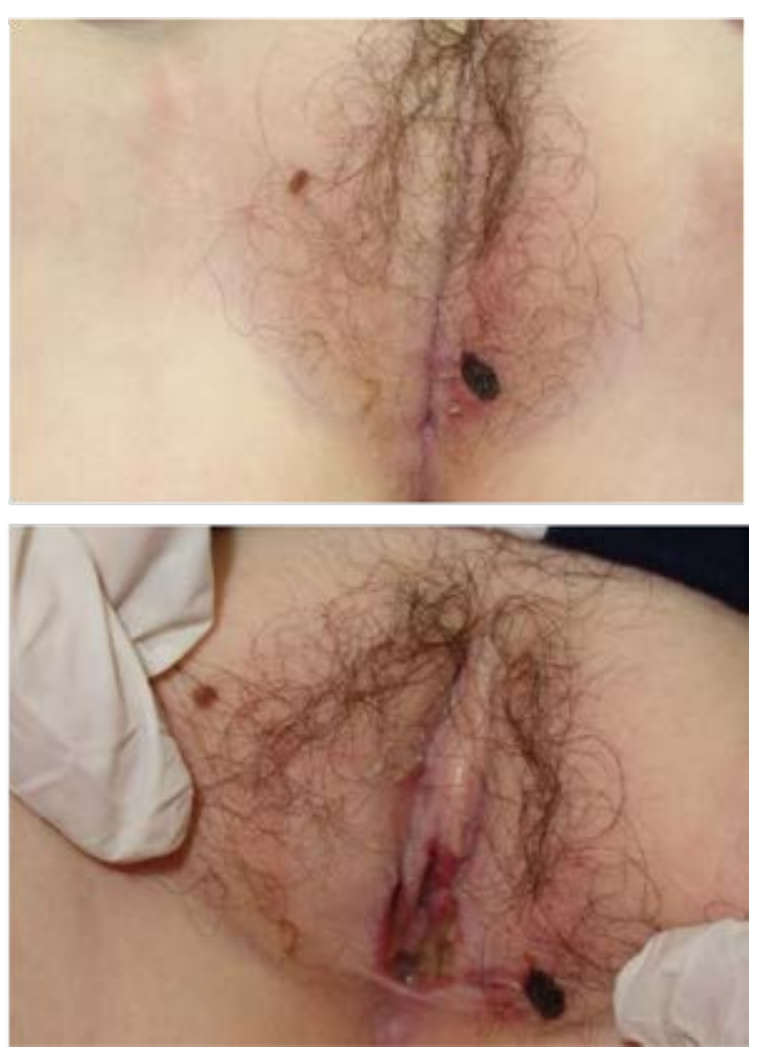

La paciente ingresó con diagnóstico de síndrome ulceroso vulvovaginal. Al momento del examen, se presentaba febril y se destacaba la presencia de úlceras necróticas vulvovaginales, dispuestas en espejo (kissing pattern) y con borde eritematoso, con secreción mucosa y placa necrótica de $2 \mathrm{~cm}$, aproximadamente, en el labio mayor izquierdo. Además, se observaba, en el labio mayor derecho, un nevo melanocítico de $1 \mathrm{~cm}$ (Figura 1).

Se realizó un estudio para el virus de inmunodeficiencia humana (VIH), sífilis por medio de venereal disease research laboratory $(V D R L)$, reacción en cadena de la polimerasa (polymerase chain reaction; $P C R$, por sus siglas en inglés) para VHS 1 y 2, PCR para Treponema pallidum, cultivo corriente y para hongos, y fueron negativos los resultados de todos los estudios mencionados.

Evaluada por Dermatología, con sospecha de úlceras de Lipschütz, se decidió realizar un estudio histopatológico de las úlceras vulvovaginales y, así, realizar el diagnóstico diferencial de una histiocitosis por las características de la lesión. La histopatología de la biopsia presentó una dermatitis perivascular intersticial mixta mononuclear y polimorfonuclear, con hemorragias recientes, focos de necrosis dérmica, hiperplasia epidérmica reactiva, sin evidencia de acción citopática viral herpética ni por virus del papiloma, ni elementos de tipo micótico, ni granulomas, ni vasculitis. La historia clínica presentada, asociada a una histopatología que descartaba otros diagnósticos, era consistente con el diagnóstico de úlceras de Lipschütz (Figura 2).

La paciente se siguió a diario durante la hospitalización y se trató con corticoides orales, prednisona a razón de $1 \mathrm{mg} / \mathrm{kg}$ en un esquema de descenso durante 2 meses, y evolucionó con reducción del dolor, de la temperatura y del tamaño de la lesión. Tuvo 2 controles ambulatorios en Dermatología, al primer y segundo mes, con la resolución completa de la lesión al mes, sin recidivas posteriores.

\section{DISCUSIÓN}

La úlcera de Lipschütz fue expuesta por primera vez en 1913, por Lipschütz, quien describió la aparición súbita de úlceras genitales dolorosas, asociadas a fiebre, linfadenopatías en mujeres adolescentes sin antecedentes de contactos sexuales. ${ }^{2}$ 
FIGURA 2. Biopsia de la piel de las úlceras vulvovaginales

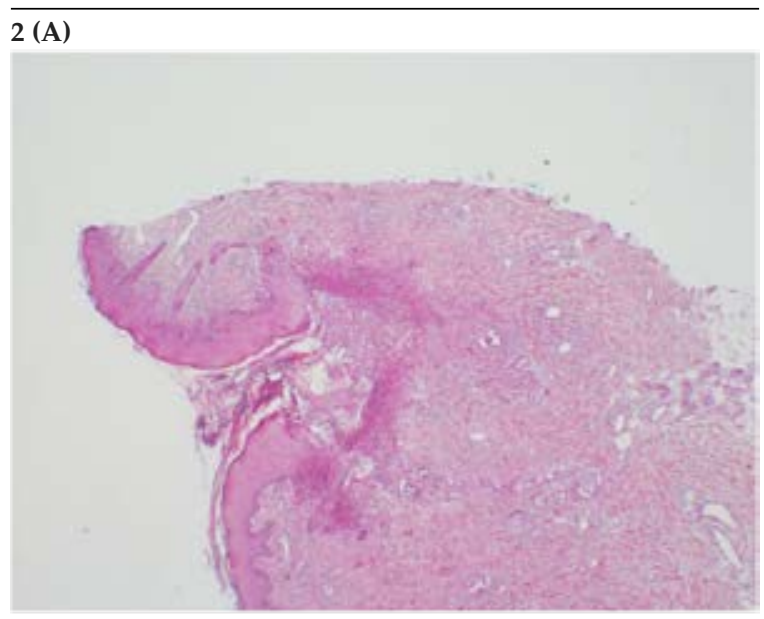

2 (B)

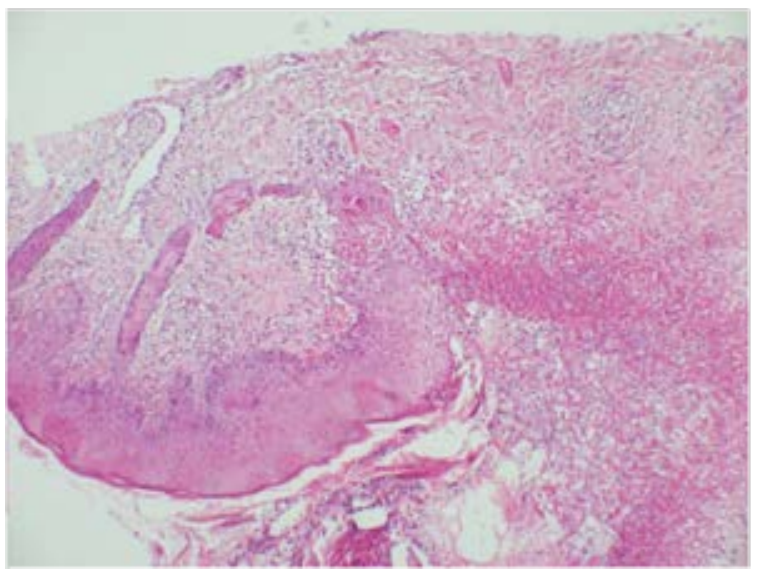

Biopsias de la piel en tinción H/E. En las imágenes con menor aumento (A), se observa una úlcera de bordes netos, constituidos por un epitelio escamoso ligeramentehiperplásico, con un lecho cubierto, en la superficie, por un exudado pseudomembranoso fibrinohemorrágico con detritus celulares. En la dermis subyacente, se observa infiltrado inflamatorio. En las imágenes con mayor aumento (B), se muestra el epitelio hiperplásico y el lecho ulceroso cubierto por fibrina y detritus celulares, con exudado inflamatorio mixto, y, en la lámina propia, dilatación vascular con infiltrado leucocitario tanto mono- como polimorfonuclear, sin signos de especificidad.

$\mathrm{H} / \mathrm{E}$ : hematoxilina/eosina.
El cuadro clínico se caracteriza por presentar una fase prodrómica, en el $50 \%$ de los casos, con cefalea, odinofagia, aftosis orales, fiebre, astenia, adinamia, anorexia, mialgias $y$, ocasionalmente, adenopatías inguinales o generalizadas..$^{2-4,10}$ Tras esto, suele presentarse gran dolor en los genitales, que precede a la aparición de la úlcera, la cual puede ser única o múltiple, con compromiso bilateral descrito en espejo o en kissing pattern, profunda, dolorosa, de base indurada, rodeada por un halo eritematoso, con edema perilesional y cubierta por una membrana necrótica. En ocasiones, se acompaña de lesiones satélites y adenopatías regionales. ${ }^{3,4,10}$

La incidencia es aún desconocida; es una enfermedad infrecuente. Tampoco se conoce su etiopatogenia ${ }^{3-5,10-15}$ y cerca del $70 \%$ son clasificadas como idiopáticas. Sin embargo, estudios recientes han demostrado una asociación con agentes infecciosos. El virus de Epstein-Barr (VEB) es el agente que se ha relacionado con más frecuencia con las úlceras de Lipschütz, ${ }^{11}$ mientras que el citomegalovirus, el virus de la influenza A, Salmonella paratyphi, ureaplasma, Toxoplasma gondii se han asociado en forma ocasional. ${ }^{5,11-14}$

La actual relación entre la úlcera de Lipschütz y diferentes agentes etiológicos no debe nublar la visión del clínico de buscar otras patologías. Por esto, ante la presencia de una erosión o úlcera genital, lo primero que se debe descartar son infecciones de transmisión sexual, otras infecciones, enfermedades sistémicas, como síndrome de Behçet, enfermedades autoinmunes, entre estas, la bullosa y el síndrome de Sjögren, y alteraciones hormonales. ${ }^{2-4,15}$

El diagnóstico de úlceras de Lipschütz se realiza por exclusión. Se establecen criterios mayores y menores para su diagnóstico en el año 2017 (Tabla 1). Son necesarios 5 criterios mayores más 1 o 2 criterios menores, los cuales deben ser

TABLA 1. Criterios diagnósticos de la úlcera de Lipschütz

\section{Criterios mayores:}

- Paciente menor de 20 años

- Presentación de un primer brote de úlcera genital aguda.

- Ausencia de contacto sexual durante los 3 meses previos.

- Comienzo abrupto y curación sin cicatriz dentro de 6 semanas.

- Ausencia de inmunodeficiencia.

\section{Criterios menores: ${ }^{*}$}

- Úlcera(s) dolorosa(s) bien delimitada(s) con un centro necrótico y/o fibrinoso.

- Patrón de beso o kissing pattern (distribución vulvar en espejo).

* Evaluación macroscópica de las lesiones ulcerosas en los genitales femeninos.

Adaptación a tabla de criterios diagnósticos. Eizaguirre F, Lucea L, Artola E, Goiri K. Úlcera genital aguda no relacionada con enfermedad de transmisión sexual. Anales de Pediatría. 2012;76(3):170-172. 
aplicados tras excluir los diagnósticos de aftosis recurrente y enfermedades de transmisión sexual, especialmente, herpes genital. Es importante tener en cuenta que esta lesión se ha asociado con el desarrollo a posteriori de enfermedades autoinmunes, como el síndrome de Sjögren, por lo que se recomienda el seguimiento de estos pacientes por la predisposición genética a respuestas inmunes a antígenos propios ante procesos inflamatorios e infecciones. ${ }^{15}$

El tratamiento consiste en el control sintomático y una adecuada higiene de la zona genital. ${ }^{2-6}$ Debe enfocarse en el alivio del dolor, para lo cual se han usado anestésicos tópicos, como lidocaína, antiinflamatorios no esteroides, corticoides tópicos y orales (sin evidencia de que modifiquen el curso de la enfermedad) y antibióticos tópicos $u$ orales de amplio espectro, aunque el curso de la enfermedad es autolimitado., Sí se ha demostrado la utilidad de antibióticos ante la sospecha de sobreinfección bacteriana y en las formas gangrenosas. ${ }^{5,10} \mathrm{La}$ utilización de baños de asiento proporciona el alivio del dolor y facilita el desprendimiento del tejido necrótico, aunque, en ocasiones, se requerirá del desbridamiento quirúrgico de este. ${ }^{2,3,5,10}$

Aún en la actualidad, esta patología se encuentra subdiagnosticada por su baja incidencia y por la falta de conocimiento, por lo que se han establecido criterios diagnósticos que facilitan el reconocimiento y el tratamiento de las úlceras de Lipschütz. Por último, es fundamental hacer un diagnóstico oportuno que permita un manejo dirigido, reforzar el carácter autolimitado de este cuadro a los padres y realizar un seguimiento cuando sea pertinente. ${ }^{2-4,15}$

\section{REFERENCIAS}

1. Gomes CM, Giraldo PC, Gomes Fde A, Amaral R, et al. Genital ulcers in women: Clinical, microbiologic and histopathologic characteristics. Braz J Infect Dis. 2007;11(2):254-60.

2. Levy Bencheton A, Agostini A, Mortier I, Sadoun C, Gamerre M. Ulcère de Lipschütz, un diagnostic mèconmu. Gynecol Obstet Fertil. 2011;39(3):e58-60.

3. Huppert JS. Lipschutz ulcers: evaluation and management of acute genital ulcers in women. Dermatol Ther. 2010;23(5):533-40.

4. Brinca A, Canelas MM, Carvalho MJ, Vieira R, Figueiredo A. Lipschütz ulcer (ulcus vulvae acutum): a rare cause of genital lesión. An Bras Dermatol. 2012;87(4):622-4.

5. Farhi D, Wendling J, Molinari E, Raynal J, et al. Non-sexualy related acute genital ulcers in 13 pubertal girls: a clinical and microbiological study. Arch Dermatol. 2009;145(1):38-45.

6. García Reymundo M, Montero Salas A, González Álvarez C, Real Terrón R. Ulcera de Lipschütz: causa poco conocida de úlcera genital aguda. An Pediatr (Barc). 2010;72(6):443-4.

7. Bandow GD. Diagnosis and management of vulvar ulcers. Dermatol Clin. 2010;28(4):753-63.

8. Rosman IS, BerkDR, Bayliss SJ, White AJ, Merritt DF. Acute genital ulcers in nonsexually active young girls: case series, review of the literature, and evaluation and management recommendations. Pediatr Dermatol. 2012;29(2):147-53.

9. Eizaguirre F, Lucea L, Artola E, Goiri K. Úlcera genital aguda no relacionada con enfermedad de transmisión sexual. An Pediatr (Barc). 2012;76(3):170-2.

10. Lehman J, Bruce A, Wetter D, Ferguson S, Rogers R 3rd. Reactive nonsexually related acute genital ulcers: Review of cases evaluated at Mayo Clinic. J Am Acad Dermatol. 2010;63(1):44-51.

11. Burguete Archel E, Ruiz Goikoetxea M, Recari Elizalde E, Beristain Rementería X, et al. Lipschütz ulcer in a 17-monthold girl: a rare manifestation of Epstein-Barr primoinfection. Eur J Pediatr. 2013;172(8):1121-3.

12. Martin JM, Godoy R, Calduch L, Villalon G, Jordá E. Lipschütz acute vulval ulcers associated with primary cytomegalovirus infection. Pediatr Dermatol. 2008;25(1):113-5.

13. Huppert JS, Gerber MA, Deitch HR, Mortensen JE, et al. Vulvar ulcers in young females: a manifestation of aphthosis. J Pediatr Adolesc Gynecol. 2006;19(3):195-204.

14. Pelletier F, Aubin F, Puzenat E, Deprez P, et al. Lipschütz genital ulceration: a rare manifestation of paratyphoid fever. Eur J Dermatol. 2003;13(3):297-8.

15. DeCastro CoelhoF, Amaral M, Correia L, Nunes Campos M, et al. Lipschütz Genital Ulceration as Initial Manifestation of Primary Sjögren's Syndrome. Case Rep Obstet Gynecol. 2018;2018:3507484. 